\title{
Perceptions and illusions of students regarding presumably undetected cheating during the COVID-19 pandemic in Greece
}

\author{
Y. Kiouvrekis ${ }^{1,3,4}$, A. Kokkinaki ${ }^{4}$ and G. Andrikopoulos ${ }^{2}$ \\ ${ }^{1}$ School of Business, Department of Business, Chios, Greece. \\ ${ }^{2}$ University of Thessaly, Larissa, Greece. \\ ${ }^{3}$ Department of Public and Integrated Health, University of Thessaly, Karditsa, \\ Greece. \\ ${ }^{4}$ Business School, University of Nicosia, Nicosia, Cyprus.
}

\begin{abstract}
The present study constitutes a preliminary research on the attitudes and perceptions of students in Greece concerning cheating in exams and plagiarism in the era of the COVID-19 pandemic. A survey conducted among 578 students shows a sharp increase in the employment of underhanded means by students towards attaining better grades in the exams. These findings need to be evaluated by the academic community to put a check on the rising number of instances of academic fraud.
\end{abstract}

\section{Keywords}

Academic integrity; Covid-19; Exam Cheating; Contract cheating;

\section{INTRODUCTION}

Academic dishonesty among University and College students is not a recent occurrence (Stephanie et al 2006); it is a phenomenon of considerable antiquity, observed throughout a range of different cultures. A large global market has developed around Universities, where firms provide services to students either in the form of tutoring or ghostwriting of term papers, senior theses, and even articles for academic journals (Clarke and Lancaster, 2013; Newton 2015; Newton 2018). The fact that in 2018 this was a 100 billion dollar market, and it is estimated to have reached 200 billion dollars in worth during the pandemic, shows that we are dealing with an intercultural practice, present in educational systems all around the globe. This is not a coincidence; during the pandemic, all Universities had to adopt remote education, which led, as previously mentioned, to an unprecedented increase in instances of academic dishonesty. The literature shows that while there have been numerous studies on this issue around the world, one is yet to be conducted in a systematic manner concerning Greece. According to Foltýnek and Králíková 2018, 8\% of Czech students have submitted a paper written by a third party as their own, $34 \%$ know someone 
who has been involved in contract cheating, and $87 \%$ are aware of firms which provide ghostwriting services for term papers and theses Reedy et al 2021 is the first study to investigate academic staff and student perceptions of online exams and forms of assessment during the COVID-19 pandemic. This study was conducted in three Australian Universities, where digital technologies had already been an integral part of University services, and it demonstrated that fraud is and has been occurring in all forms of online exams. It concludes that continued research is necessary to determine how to better ensure academic integrity in online exams in the future.

The COVID-19 pandemic forced a violent readjustment of educational practice, with higher education institutions globally adopting a hybrid mode of education. Our case study focuses on Greece, as in this country, only a single institution among all Greek Universities had been offering e-learning education programs in place prior to the advent of the pandemic.

This lack in infrastructure and experience on how to plan and implement assessment of students who followed online or hybrid courses led to egregious instances of breaches of academic integrity (Abukari, 2016). During all exam periods in 2020 and 2021, systematic attempts of students to cheat have been identified ranging from inventive ways to gain access to course content to posts in social media ads asking for private tutors who would be willing to impersonate students in the exams and take them in their stead. An attempt has been made to examine the extent and impact of such actions as well as to document student perceptions on issues of academic integrity and scientific/research ethics.

Literature suggests that student perceptions on what constitutes breaches of academic integrity may differ according to their educational background and progress in their studies (Amigud et al., 2019). Violations of academic integrity -i.e. students cheating on exams in the context of a sudden transition from live to remote education - constitute a grave issue and reveal a failure on behalf of faculty to impart scientific principles to students (Stella-Maris et al, 2017).

Recent publications suggest that breaches of academic integrity have seen an increase in Universities of both developed and developing countries - certainly a salient finding, as it highlights the causes which recently led to an increase in attempted exam cheating or contract cheating in technologically advanced countries like Greece (Ellis et al., 2018).

Given that in order to address any issue, one needs to fully comprehend its nature, the present study focuses on how a sample of Greek students interprets academic integrity. There are significant differences not only across disciplines and institutions, but also in the ways individual students and members of 
faculty understand and employ basic terms as well as in the actions they take towards preventive or corrective measures, or the excessive quotation of source texts. To this end, we designed an original questionnaire, taking into consideration the most common instances of academic integrity violations. The survey revealed a marked discrepancy on the one hand between expectations set by Universities and faculty, and on the other hand the manner in which students perceive what constitutes proper academic conduct of their studies. Students' perceptions as well as their illusions are highlighted and discussed.

The topic examined in this context, although it focuses on findings from data analysis from a specific area, is actually relevant to academic settings in many other countries (Khomami, 2017; Marsh, 2017). The research presented focuses on the analysis of breaches of academic integrity during exams from the perspective of students. The main research objectives are listed below:

1. Collect data relevant to breaches of academic integrity in higher education in Greece during the pandemic period.

2. Identify possible factors motivating students to breach academic integrity.

3. Compare findings in relation to other similar surveys.

Our methodological approach is based on qualitative research (qualitative and non-numerical data) as well as quantitative research (quantitative or numerical data). Therefore for this study, the methodology chosen is that of the mixed method - we designed a survey that would collect data for both quantitative and qualitative study. The survey was conducted based on a questionnaire to make a systematic investigation of the characteristics, opinions and the relationship between the variables of the questionnaire. The sampling method chosen is group sampling, with a sample of 578 students.

\section{FINDINGS AND DISCUSSION}

Data collection was conducted through a survey, which took place online (using Google forms) from April, $1^{\text {st }}$ to May $31^{\text {st }}$ 2021. It was targeting students at Universities, technical universities and other tertiary education institutes in Greece. Convenience sampling method was selected and a total of 578 valid and complete questionnaires were received from nine different Universities in Greece. Participating Universities include four from major, metropolitan areas of Greece, while the remaining cover the remaining mainland and islands of Greece.

Among participants, $63.4 \%$ identified themselves as "Female" and $36.2 \%$ as "Male". Responders came from the three different cycles of tertiary education, 
namely $77.9 \%$ were undergraduate students, $19.7 \%$ followed postgraduate studies (MSc or MA programme) and 2.2\% were doctoral students.

When asked to identify the main motivation for attending the University, $39.5 \%$ stated that their main aim was to acquire new knowledge and skills, while $35.7 \%$ stated they wanted to acquire a formal qualification and $20.7 \%$ stated their studies was a means to advance their careers. The most cited motivation for attending University is in alignment with the mainstream culture in Greek society, where families go through extreme financial sacrifices to prepare their children through private tutoring to take the competitive entrance examinations and secure a position at the University.

The majority of responders (52.8\%) identified their academic performance as adequately good, whereas $26.6 \%$ of them proclaimed that their performance is very good, and $20.6 \%$ of students claimed that their performance is average.

An overwhelming $81.3 \%$ declared that they were familiar with contract cheating services, and their awareness was raised primarily through social media (43.2\%) and through word of mouth (32.6\%). Furthermore, it appears to be very rare that students ask from a family member, a friend or an acquaintance assistance in completing an assignment; 89.9\% responded they have never requested it while the remaining $11.1 \%$ admitted that they had.

More specifically, for those students who have used contract cheating, the majority $(63.4 \%)$ admitted that they had paid in exchange of the provided assistance, while the remaining $36.7 \%$ had received help for free, presumably from some acquaintance.

It is interesting to see whom they solicited to contract cheating. The leading answer $(31.3 \%)$ involves "a friend" followed by $18.8 \%$ of students who assigned the task to an academic private tutoring service; $14.6 \%$ convinced a fellow student to do it, and $10.4 \%$ employed the services of a private tutor/professional specialist. As an explanation for this act, $29.1 \%$ blamed it on the lack of time, $27.9 \%$ on the fact that they were overwhelmed with too many assignments having deadlines close one to the other, $16.3 \%$ were afraid of failure, and $12.8 \%$ had no confidence in their own ability to complete the assignment. The majority of students surveyed $(51.7 \%)$ claimed that they edited the assignment themselves before submitting it, $21.8 \%$ submitted it exactly as it had been received, whereas the remaining $11.5 \%$ ended up not submitting it at all.

The overwhelming majority of students (90.9) is aware that that commissioning a written assignment to a third party is a form of academic dishonesty. It must be noted that in $96.1 \%$ of cases of contract cheating the faculty member did not 
find out that the submitted assignment was the result of contract cheating. Furthermore, even when the student was confronted, $95.8 \%$ of students did not face any penalty.

In an attempt to understand the extent of this phenomenon, students were asked if they were aware of any friends or fellow students who had been involved in contract cheating at some point; $68.3 \%$ of students responded that indeed they were aware of at least one case. Furthermore, $73.9 \%$ of responders believe that the pandemic contributed to the increase of academic dishonesty. This position is supported by the fact that those responders who admitted that they did use private tutoring support in their assignment had not resorted (by almost $74 \%$ ) to such practices before the onset of the pandemic. Moreover, the majority $(59 \%)$ of students who did undertake contract cheating believed that such practices had seen an increase since the beginning of the pandemic.

Another topic of concern is the organization and conducting of final exams. During the pandemic, all Greek Universities had to hold remote exams during the examination periods in Spring and Fall 2020 and in Spring 2021. Most Universities plan to hold face-to-face exams in Fall 2021. Students' perceptions of the organizational aspects for remote exams is almost evenly split; $51 \%$ of them thought they were not properly organized while the rest regarded them as well organized. Apart from cheating in its various forms, the final examinations seem to face another issue of the highest severity. A slim percentage of $5.6 \%$ of students admitted that they have asked another person to take the exam in their stead; furthermore, $31.5 \%$ of students claimed they were aware of sites or establishments that offer such services.

\section{CONCLUSIVE REMARKS}

The research in this paper is focusing on breaches of academic integrity from the viewpoint of students in Greek Academia. Two phenomena appear to be officially reported for the first time in this paper. The first concerns the extent of contract cheating in Greek tertiary education. It appears that the extent of contract cheating is considerable and it is mostly carried out by professional services rather than contributions from friends, family members or acquaintances. Contract cheating is usually associated with assignments that carry significant weight towards final grading. The most cited reasons for doing it are not uncommon: time shortage and an overwhelming number of assignments are usually blamed. The other major finding is identifying that impersonation of a student during final examinations by someone else who is an expert on the topics takes place in at least about $5 \%$ of examinees. The exact percentage of this particular kind of academic dishonesty needs to be investigated further; however, preliminary evidence points to the fact that a 
shadow ecosystem of impersonators has been evolving and it is evolving during the pandemic with remote examinations.

\section{References}

Abukari, Z. (2016). Awareness and Incidence of Plagiarism Among Students of Higher Education: A Case Study of Narh-Bita College. Doctoral Dissertation, University of Ghana.

Amigud, A., and Lancaster, T. (2019). 246 reasons to cheat: an analysis of students' reasons for seeking to outsource academic work. Compu. Educ. 134, 98-107. doi: 10.1016/j.compedu.2019.01.017

Clarke, R., and Lancaster, T. (2013). "Commercial aspects of contract cheating,"in Proceedings of the $18^{\text {th }}$ ACM Conference on Innovation and Technology in Computer Science Education, (New York, NY: ITiCSE'13; ACM), 219-224. ,

Ellis, C., Zucker, I.M. \& Randall, D. The infernal business of contract cheating: understanding the business processes and models of academic custom writing sites. Int J Educ Integr 14, 1 (2018). https://doi.org/10.1007/s40979-017$\underline{0024-3}$

Foltýnek, T., \& Králíková, V. (2018). Analysis of the contract cheating market in Czechia. International Journal for Educational Integrity, 14, 1-15.

Khomami, N (2017). Plan to crack down on websites selling essays to students announced. Higher Education. The Guardian. URL.

https://www.theguardian.com/education/2017/feb/21/plan-to-crack-down-onwebsites-selling-essays-to-students-announced

Newton, D. (2015). Cheating in Online Classes is Now Big Business. Boston, MA: The Atlantic, 4

Newton, P. (2018). How common is commercial contract cheating in highereducation? Front. Educ. 3, 1-18. Doi: 10.3389/fedue.2018.00067

Marsh, S. (2017). Universities urged to block essay-mill sites in plagiarism crackdown. The Guardian: Higher Education 
https://www.theguardian.com/education/2017/oct/09/universities-urged-toblock-essay-mill-sites-in-plagiarism-crackdown.

Reedy, A., Pfitzner, D., Rook, L. et al. Responding to the COVID-19 emergency: student and academic staff perceptions of academic integrity in the transition to online exams at three Australian universities. Int J Educ Integr 17, 9 (2021). https://doi.org/10.1007/s40979-021-00075-9

Rogerson, A. (2018). Detecting contract cheating in essay and report submissions: process, patterns, clues and conversations. In International Journal for Educational Integrity 13(10). Open: Springer.

Stephanie Etter, Jackie J. Cramer \& Seth Finn (2006) Origins of Academic Dishonesty, Journal of Research on Technology in Education, 39:2, 133-155, DOI: 10.1080/15391523.2006.10782477

Stella-Maris, O., and Awala-Ale, A. (2017). Exploring students' perception and experience of ghostwriting and contract cheating in Nigeria higher education institutions. World J. Educ. Res. 4:551. doi: 10.22158/wjer.v4n4p551 